\title{
KONJEKTURVORSCHLÄGE ZU HYMNEN DES ROMANOS MELODOS
}

Günter Prinzing zum 60. Geburtstag

Die Auseinandersetzung mit den Problemen der im Folgenden behandelten Textstellen bei Romanos Melodos ${ }^{1}$ ergab sich im Zusammenhang mit der Übersetzung einiger Hymnen in die deutsche Sprache. Den Textstellen ist gemeinsam, dass sie, ausgenommen die unter den Nummern 3 und 13 diskutierten, nur im sogenannten Patmos-Kontakar des 11. Jahrhunderts überliefert sind, den Codices Patmiaci 212 und 213 (P und Q) ${ }^{2}$.

\section{Auf Adam und Eva (H. 1, Grosdidier de Matons, H. 51, Maas / Trypanis)} In diesem Hymnus lautet das Ende der 17. Strophe in Codex Q:

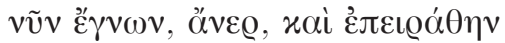

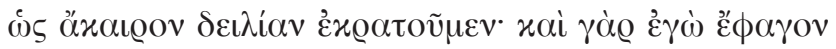

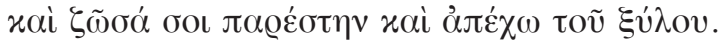

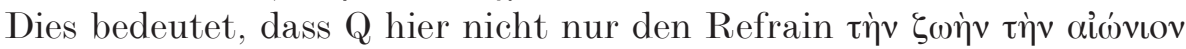
weglässt, sondern zudem von dem metrischen Schema der letzten Zeile im Oikos (es lautet inklusive des Refrains: - ' . . ' _ . ' _ . ' . ' - . ) erheblich abweicht.

${ }^{1}$ Zitiert werden die folgenden Textstellen nach J. Grosdidier de Matons, Romanos le Mélode, Hymnes, I-V (SC 99/110/114/128/283). Paris 1964-1981. Vergleichend werden auch die Ausgabe von P. MaAs und C.A. Trypanis, Sancti Romani Melodi Cantica. Cantica Genuina. Oxford 1963, und Cantica Dubia. Berlin 1970, sowie die Ausgaben

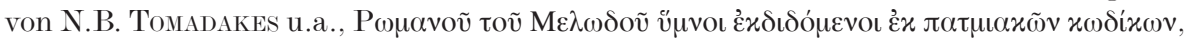
I-IV. Athen 1952-1961, und die von einer italienischen Übersetzung begleitete von R. Maisano, Romano il Melode, Cantici (Classici Greci, Autori della tarda antichità e dell'età bizantina), I-II. Turin 2002, herangezogen.

${ }^{2}$ Die Datierung der beiden Handschriften scheint unstrittig zu sein, vgl. J. Grosdidier de Matons, Romanos le Mélode et les origines de la poésie religieuse à Byzance. Paris 1977, 70, sowie die Einleitungen der genannten Ausgaben Grosdidier de Matons I 28f., Maas / Trypanis XXVf., und Tomadakes 2.1 (ausführlich: M. Naumides, S. $\varepsilon-\sigma \mu \delta$, und P. Nikolopulos, S. $\sigma v \theta-\tau v \theta)$. - Auf die besondere Problematik des Patmos-Kontakars weist H. Hunger in seiner Rezension der Edition von Grosdidier de Matons, BZ 61 (1968) 90-96, hin. 
Die Herausgeber Maas / Trypanis und Grosdidier de Matons erkannten,

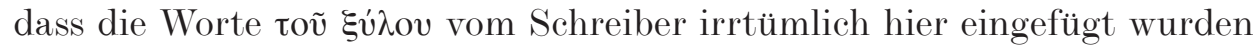
und dass er sie aus der entsprechenden Zeile der vorangehenden 16. Strophe übernahm ${ }^{3}$. Daher eliminierten sie die beiden Worte und fügten den Refrain hinzu (wodurch die Zeile auch metrisch geheilt wurde):

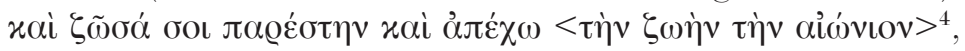
was Grosdidier de Matons mit „et je suis là près de toi, bien en vie, et j'en recueille <la vie éternelle $>$ “ und Maisano mit „eppure sono viva qui accanto a te e possiedo la vita eterna" wiedergeben.

Inhaltlich geht es hier darum, daß Eva bereits - vermeintlich unbescha-

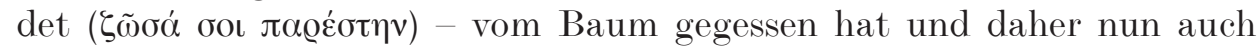
Adam von der Frucht anbietet, die nach den Worten der Schlange (Strophe 14, Z. 6) gottgleich macht. In diesem Aussagezusammenhang passt die bei Romanos sonst zweimal (H. 1.7.2 und 52.20.8) belegte Bedeutung von

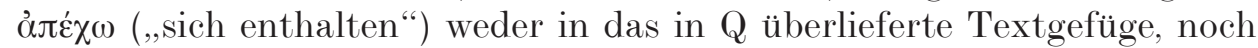
in das von den Herausgebern rekonstruierte. Daher schlage ich vor, zwar den Text im übrigen in der von Maas / Trypanis und Grosdidier de Matons

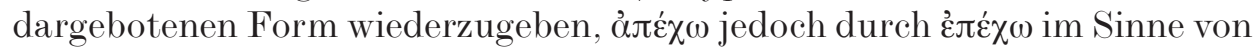
„etwas behalten“, „festhalten an etwas“ zu ersetzen. Dieser Konjekturvor-

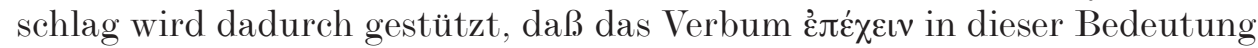
bei Romanos auch an einer weiteren Stelle, im Hymnus „Auf alle Märtyrer" ${ }^{\text {“5 }}$ begegnet. (An zwei weiteren Stellen wird es im Sinne von ,zurückhalten“ bzw. ,behindern“ gebraucht ${ }^{6}$.)

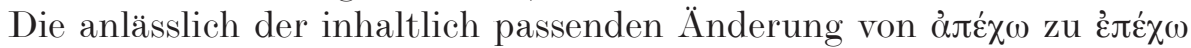
vermutete Verlesung von $\varepsilon$ als $\alpha$ ist in einer Minuskelvorlage des Codex Q im Zeitraum zwischen dem 8. und dem 11. Jahrhundert paläographisch nachvollziehbar ${ }^{7}$. Dies gilt speziell in der Ligatur mit folgendem $\pi$ am Wortbeginn ${ }^{8}$.

3 Dort lautet nämlich die letzte Zeile:

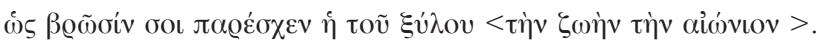

${ }^{4}$ Grosdidier de Matons, H. 1.17, Z. 92 (mit erklärender Fußnote), Maas / Trypanis, H.

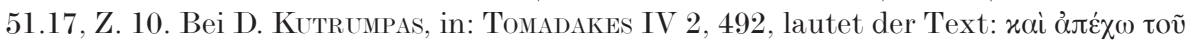

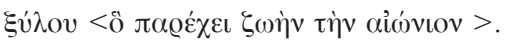

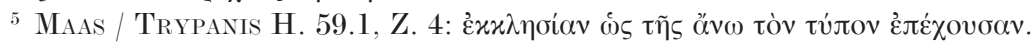

${ }^{6}$ Grosdidier de Matons H. 3.12.6 (,zurückhalten“) und H. 39.16.10 (,,behindern“); vgl. auch MaAs / Trypanis H. 61.16.8 (,,behindern“).

7 Es dürfte kein Zufall sein, dass in der selben Strophe (H. 1.17.5) das von Q tradierte

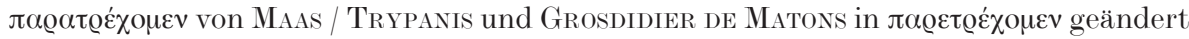
wurde.

8 Vgl. etwa bei E. Foldieri, Codices graeci Bibliothecae Vaticanae selecti (Exempla scripturarum, 4). Vatikanstadt 1969, auf den Tafeln 12-22 (Codices zwischen dem Beginn 
Man kann den Text von Hymnus 1.17.7-9 dann folgendermaßen verstehen:

Jetzt habe ich erkannt und erfahren, Mann, $d a \beta$ unnötige Furcht uns befangen hielt. Denn ich aß davon und trat doch lebend vor dich hin und halte fest am ewigen Leben.

\section{Auf Joseph (H. 5, Grosdidier de Matons, H. 43, Maas / Trypanis)}

Die Brüder des Joseph suchen ihn in Ägypten auf, um eine Getreidespende zu erlangen. Nicht wissend, dass sie vor ihrem Bruder stehen, sagen sie zu ihm über ihren jüngsten Bruder Benjamin, laut Codex Q (19.8):

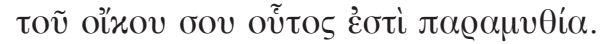

Dieser Vers enthält metrische und inhaltliche Probleme. Die metrische Korruptel heilte Grosdidier de Matons, indem er der handschriftlich tradierten Form $<v \tilde{v} v>$ voranstellte ${ }^{9}$ und so - zudem unter Verdeutlichung des inhaltlichen Zusammenhanges - die korrekte Versform bewirkte: - - ' - '

-. Ich würde (unter Weglassung von oṽ̃os, s. unten) anstelle von vข̃v eher ôs vorziehen, was bei gleich bleibenden metrischen Folgen einen besseren Anschluss an den vorangehenden Vers bewirkt.

Eine inhaltliche Schwierigkeit ergibt sich dadurch, dass mit oov ja Joseph gemeint sein muss und die (tatsächlich gegebene) Blutsverwandtschaft an dieser Stelle des Hymnus noch als unerkannt zu gelten hat, es sei denn, man folgt der Überlegung Grosdidier de Matons (dem dann auch Maisano zu folgen scheint), dass man infolge eines Sklavenverhältnisses der Brüder und des Vaters zu Joseph von einer bestehenden familiären bzw. „häuslichen" Zusammengehörigkeit sprechen kann. Dies erscheint mir zu weit hergeholt. Krumbacher, der das Problem ebenfalls sah, korrigierte in: toṽ

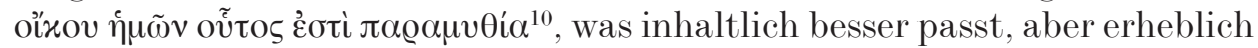
gegen des Metrum verstößt. Daher schlage ich vor, das oov oṽ̃os in toṽ $\pi \alpha$ ¿ò zu verbessern $^{11}$. Der Vater - er wird zuvor in Vers 3 genannt - ist ja der eigentlich durch Benjamin Getröstete, nicht die Brüder. Die metrische Abweichung, die sich durch $\pi \alpha$ toò auch in Oikos 33 des selben Hymnus.

des 9. und dem Ende des 10. Jahrhunderts) die entsprechenden Ligaturen.- Für freundliche Hilfe bei paläographischen Fragen danke ich Ernst Gamillscheg, Wien.

${ }^{9} \mathrm{Ihm}$ schließt sich Maisano II 227, A. 41, an.

${ }^{10}$ K. Krumbacher, Studien zu Romanos. Sb. ph.h. Kl. Bayer. AW 1898, 2/1, 69-268, der Hymnus 135ff., zur Stelle: 148.

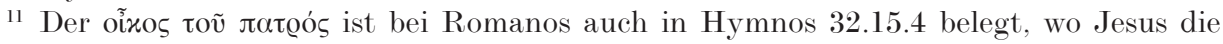
Händler aus dem Haus des Vaters (dem Tempel in Jerusalem) vertreibt. 
Text und Übersetzung der Verse 7 f. könnten dann lauten:

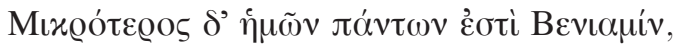

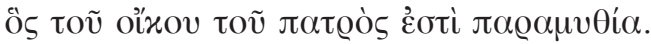

Der jüngste von uns allen ist Benjamin,

der Trost im Hause meines Vaters.

\section{Stichera auf die Geburt Christi (H. 13, Grosdidier de Matons, H. 83, Maas / Trypanis)}

Der Hymnus ist lediglich in den Codices Vallicellianus E 54 (l, s. XI), Vat. gr. 1212 (m, s. XII, nur Oikos 1-7) und Vat. gr. 1531 (o, s. XV/XVI) erhalten. Er feiert den Neugeborenen in der Krippe. Oikos 14.1f. besingt die gleichzeitige Gegenwart Christi in der Krippe und im Himmel, zur Seite des Vaters:

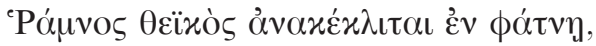

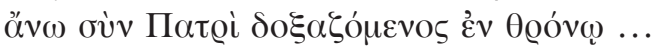

Oikos 17 greift den Gedanken im Zusammenhang mit der übernatürlichen Empfängnis der Mutter Gottes erneut auf; 17.3f. lautet (in Codex l):

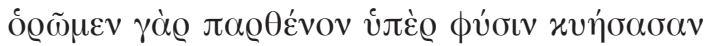

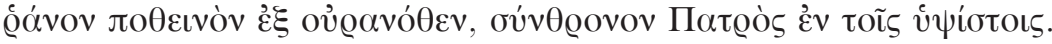

Grosdidier de Matons und Maisano („Il divino roveto ..."12) akzeptierten in Oikos 14.1 das Wort @ó́uvos („Kreuzdorn“) und führten es in Oikos 17.4 durch Verbesserung aus der handschriftlichen Form @óvov ein, wenngleich Grosdidier das Bild als „sehr bizarr" erschien ${ }^{13}$ - dies zu Recht, denn in den Textstellen der Bibel, die das Wort verwenden ${ }^{14}$, ist kein sinnvoller Zusammenhang mit dem Inhalt des Hymnus zu erkennen.

Das Wort @óuvos wurde bereits von Pitra als unpassend empfunden, der

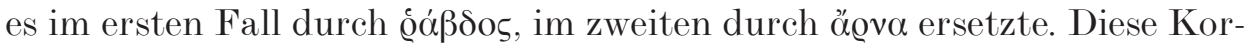
rekturen erscheinen mir nicht zutreffend, da @óßjos (der Stamm Jesse) ja gewissermaßen die Elternrolle hat, aus der Christus (in 17.1 passend als őv 0 os tituliert) sprießt; daher liegt er eben nicht in der Krippe.

Daher schlage ich in beiden Fällen die einfache Verbesserung zu ỏuvós („Lamm“) vor, die inhaltlich passend ist ${ }^{15}$ und metrisch insoferne unproblematisch, als der Akzentwechsel von Paroxytonese zu Oxytonese inner-

12 Maisano II 559, mit A. 22, in der unter Verweis auf Ex 3.2 darauf verwiesen wird, dass der brennende Dornbusch zwar meist als Bild Mariens gesehen werde, fallweise jedoch auch die beiden Naturen Christi symbolisiere.

13 Grosdidier de Matons II 148f., mit app. crit. und A. 1, 150f. mit app. crit. und A. 1.

14 Jud 9.14-15 (Jotams Fabel), Ps 57.10 (Gericht über die irdischen Richter).

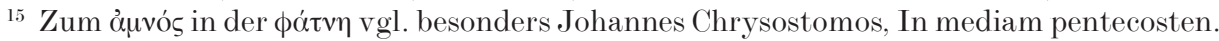
$P G 61.743 \mathrm{D} / 744 \mathrm{~A}$. 
halb des Hymnus auch an anderer Stelle nachweisbar ist ${ }^{16}$. Die Übersetzung der beiden Textstellen lautet dann:

Oikos 14.1f.:

Das göttliche Lamm liegt in der Krippe

und wird oben auf dem Thron mit dem Vater gepriesen.

Oikos 17.3f.:

Denn wir sehen eine Jungfrau, die das ersehnte Lamm

übernatürlich empfangen hat, vom Himmel, wo es mit dem Vater in der Höhe thront.

\section{Auf die unschuldigen Kinder (H. 15, Grosdidier de Matons, H. 3, Maas / Trypanis)}

In diesem Hymnus weigern sich die Soldaten des Herodes zunächst, in Bethlehem alle Kinder zu töten, sind aber bereit, die Stadt zu durchsuchen, und sie versichern dem Herodes laut Codex P (5.11f.):

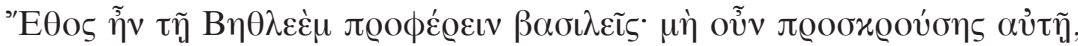

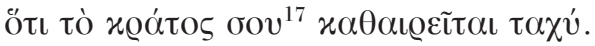

Die Bedeutung des vorletzten Verses widerspricht inhaltlich dem Refrain und lässt die Aussagen der Soldaten unlogisch erscheinen, da diese sich ja auch im weiteren Verlauf des Hymnus gegenüber Herodes loyal

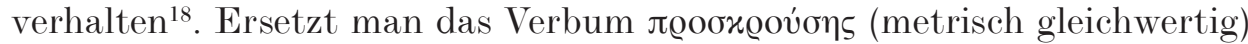
durch $\pi$ $\tau_{\varepsilon v ́ \sigma \eta \varsigma^{19}}$, dann ergibt sich folgende, inhaltlich widerspruchsfreie Übersetzung:

Es war Bethlehems Gewohnheit, Könige hervorzubringen; daher glaube ihr nicht,

dass deine Macht bald zuschanden wird!

\section{Auf die unschuldigen Kinder (H. 15, Grosdidier de Matons, H. 3, Maas / Trypanis)}

Im selben Hymnus widerspricht der Refrain am Ende der neunten Strophe inhaltlich dem Kontext (9.10-12):

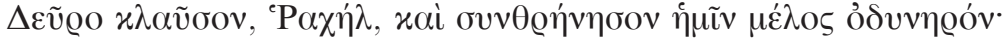

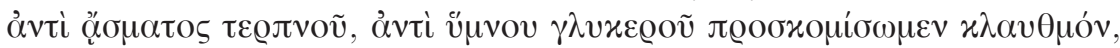

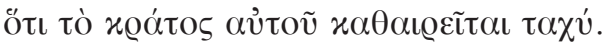

${ }^{16} \mathrm{Zu}$ Oikos 14.1 vgl. 2.1, 7.1, 9.1, 10.1, 30.1, 32.1, zu Oikos 17.4 vgl. 10.4, 11.4.

17 бov: tò oóv MaISANo I 152.

18 Der Widersprüchlichkeit des Textes waren sich auch Grosdidier de Matons II 211 , A. 2, und Maisano I 153 A. 15, bewusst.

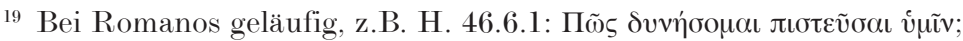


Denn Rachel würde dann ja aufgefordert, gemeinsam mit den ermordeten Kindern von Bethlehem darüber zu klagen, „dass des Herodes Macht bald zuschanden wird" 20 .

Nun ist der Refrain in diesem Hymnus nicht einheitlich; er passt sich vielmehr jeweils dem Sinn der Strophen an. Folgende Varianten begegnen:

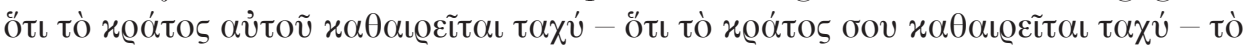

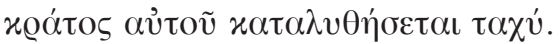

Ersetzt man nun beim Refrain das erste Wort őtı durch ö $\mu \omega s$, dann ergibt sich ein zutreffender Sinn, da im Refrain auch diesfalls gewissermaBen ein Ende des Leidens angekündigt wird. Der Ersatz von őtı durch ő $\mu \omega \varsigma$ verändert das Metrum nicht; ő $\mu \omega \varsigma$ wird zwar meist nachgesetzt, doch ist es auch an erster Stelle möglich, insbesondere bei Romanos ${ }^{21}$.

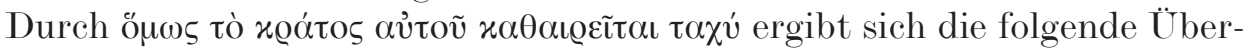
setzung:

Hierher, Rachel, weine und wehklage mit uns in einem Trauerlied!

Anstelle eines Freudengesanges, anstelle eines süßen Hymnus wollen wir eine Klage anstimmen!

Doch bald wird seine Macht zuschanden.

\section{Auf die Samaritanerin (H. 19, Grosdidier de Matons, H. 9, Maas / Trypanis)}

In diesem Hymnus lautet der Beginn der 13. Strophe in Codex Q (13.1-3):

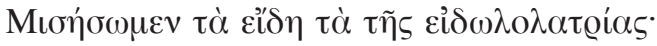

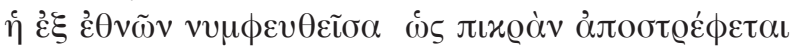

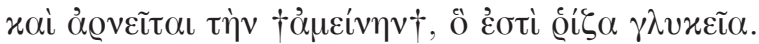

Grosdidier de Matons ${ }^{22}$ stimmt Maas / Trypanis zu, die zu dieser - metrisch nicht auffälligen - Textstelle „ỏukiv ๆv non intellegitur" vermerken, und verweist auf den (vermutlich pseudepigraphen) Hymnus „Auf den hl.

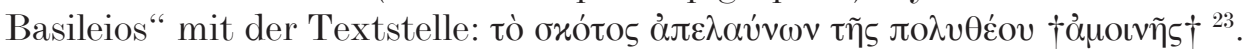
Am Rande erwägt (und verwirft) er weiters einen etymologischen Zusam-

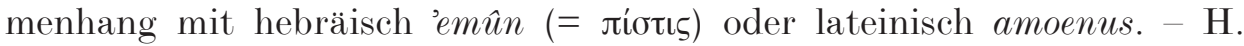

${ }^{20}$ Der Widersprüchlichkeit des Textes waren sich auch Tomadakes, Grosdidier de Matons II 217, A. 1, und Maisano I 157 A. 29, bewusst. Ersterer änderte den Text zu x@óto

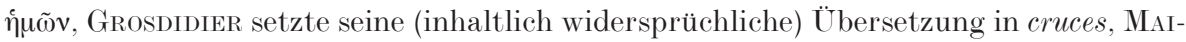
sano übernahm den Text von Grosdidier, übersetzte jedoch inhaltlich sinnvoll (und meinem Konjekturvorschlag entsprechend): „Ma il suo potere sarà presto annientato".

${ }^{21}$ Vgl. LSJ 1230a, sowie Romanos, H. 1.2.7, 8.5.5, 8.12.3, 8.19.3, 9.7.7, 17.6.3 et passim.

${ }^{22}$ Ebd. II 342f. A. 1.

${ }^{23}$ H. 82 (Maas / Trypanis 86).2, Z. 2. 


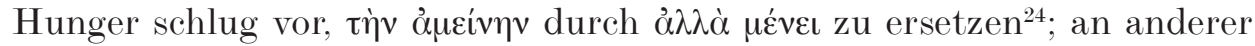
Stelle sprach er (mit Grosdidier) von einer ,gut schmeckenden, aber giftigen

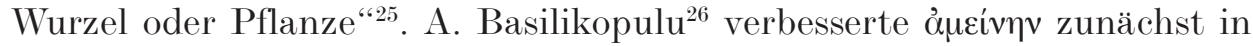

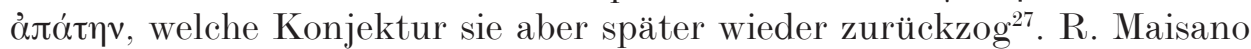

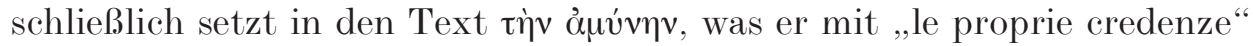
übersetzt ${ }^{28}$.

Betrachtet man den Kontext, so stellt man fest, dass Romanos in der vorangehenden zwölften Strophe die Samariterin, die sich fünf Männern hingegeben hatte und nun keinen Mann hat, mit der Kirche vergleicht, die fünf Hauptfehler unter den zahlreichen Irrtümern der Götzenanbeter ver-

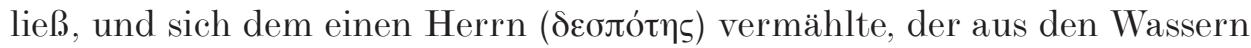

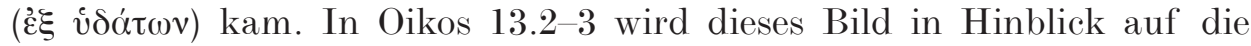
Samariterin weiter entwickelt: Sie lehne nun die $\dagger \dot{\alpha} \mu \varepsilon i v \eta v+$ als bitter ab, obwohl dies doch eine süße Wurzel sei ${ }^{29}$. Das Bild der bitteren Wurzel, die „,alle vergiftet“, entstammt dem Hebräerbrief (12.15) bzw. dem Deuteronomion (29.17); Romanos bezog sich vielleicht auch auf eine Passage bei Johannes Chrysostomos ${ }^{30}$.

Welche textnahen Verbesserungsmöglichkeiten bieten sich an? Roma-

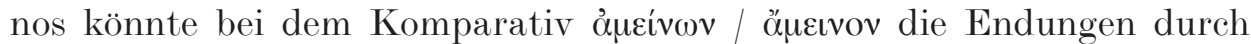

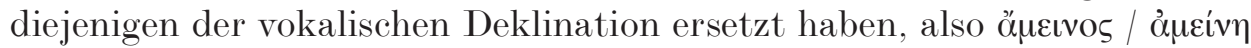

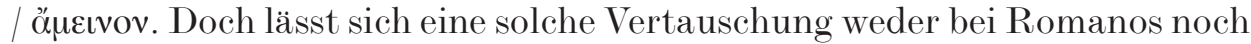
bei anderen frühbyzantinischen Autoren nachweisen ${ }^{31}$, weshalb sie unwahrscheinlich ist. Eine orthographisch und lautlich naheliegende Konjektur würde zu dem Substantiv ỏ uv́vๆv führen, das zweimal bei Johannes Lydos belegt ist ${ }^{32}$, doch ist diese Erklärungsmöglichkeit wegen der inhaltlichen Sinnlosigkeit ihrer Grundbedeutungen (,Verteidigung, Abwehr“) zu ver-

${ }^{24}$ In seiner Rezension in BZ 57 (1964) 440.

25 In seiner Rezension in $B Z 61$ (1968) 94.

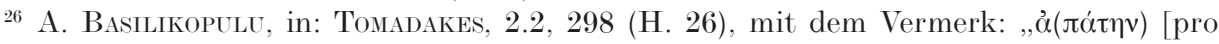
$\pi \lambda \alpha ́ v \eta$ v. 229] Tomadakis supponet".

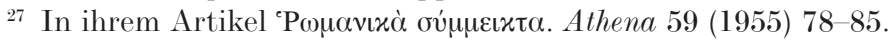

28 Maisano I 262f.; vgl unten, A. 33.

29 Der bildhafte Gebrauch des Kontrastes süß / bitter, im Zusammenhang mit Pflanzen und Wurzeln begegnet bei Romanos häufig, vgl. bes. H. 6.5.3f. (

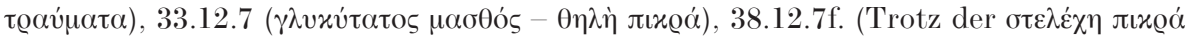

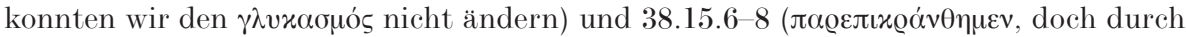
das Kreuz wurde die Erde $\gamma \lambda$ $\lambda \varepsilon \varepsilon \tilde{\alpha} \alpha)$.

30 In epistulam ad Hebraeos, PG 63.213CD.

31 Überprüft anhand der Lexika LSJ, LAмpe und LBG, sowie des TLG.

32 TLG E: Johannes Lydos, De mag. 242.3, 252.11, demgegenüber ǒ́uvav passim, einmal auch bei Johannes Lydos: Ost. 48.15. 
werfen $^{33}$. Weiters findet sich in einem Galenischen Traktat die Beschreibung eines heilsamen Krautes namens $\alpha \mu^{34}$, das unter anderem als $\gamma \varepsilon v o-$

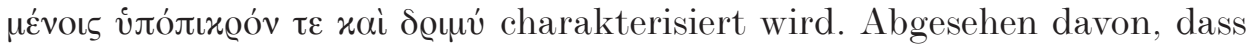
dieses Kraut tatsächlich bitter ist (während die Samaritanerin ein vermeintlich bitteres zurückweist), würde der Akkusativ őjuv ein metrisches Problem schaffen.

Sucht man nach Alternativen, die mit „süßen Wurzeln“ in einen Zusammenhang zu bringen sind, so findet man einen Hinweis auf eine süße Pflanze im Kommentar des Eustathios von Thessalonike zur Geographie des Dionysios Periegetes. Hier heisst es in einem Bericht über ein Zucker-

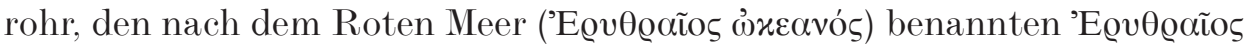

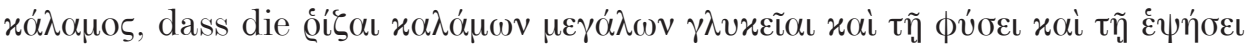

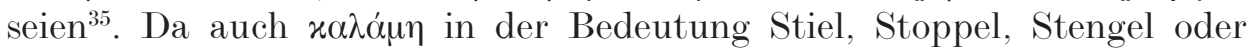

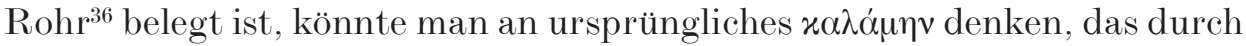

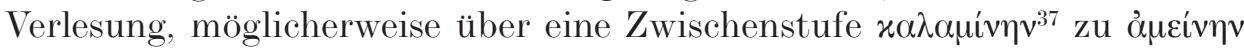
wurde. Doch ist zu bedenken, dass $x \alpha \lambda \alpha \dot{\mu \eta}$ (und xó $\lambda \alpha \mu \mathrm{s}$ ) im engeren biblischen Textumfeld nicht als „süße Wurzel“ belegt ist, sondern (meist in negativem Symbolzusammenhang) als „Stroh", „schwankendes Schilfrohr" oder „,(zerbrochener) Rohrstab“, weiters auch „Schreibrohr“ und „Maß$\mathrm{stab}^{\text {"38 }}$. Auch bietet sich eine gedankliche Verbindung zur Schwiegermutter Ruths im Alten Testament an, die nicht mehr Noemi heissen will, sondern Marah (Пıю@́, die „Bittere“) ${ }^{39}$, doch wäre diesfalls sowohl der Sinnzusammenhang als auch die sprachliche Texteinbindung schwierig.

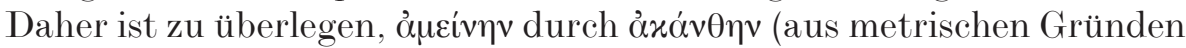

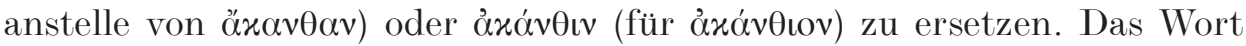
är $\alpha v \theta \alpha$ und davon abgeleitete Wörter sind bei Romanos oft belegt ${ }^{40}$, darunter auch in inhaltlich vergleichbaren Zusammenhängen ${ }^{41}$. Dies gilt aller-

${ }^{33}$ Daher erklärte auch Maisano 263, A. 46, seine Übersetzung, die auf einer belegten Sonderbedeutung von åuv́vฑ beruht.

${ }^{34}$ De simplicium medicamentorum temperamentis ac facultatibus, unter dem Lemma $x \eta^{2}$.

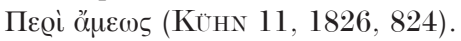

${ }^{35}$ Eustathios von Thessalonike, In Dion. Per. 1107.40ff. Die Suche erfolgte mit Hilfe von TLG E.

${ }^{36}$ LSJ 865ab, F. Preisigke, Wörterbuch der griechischen Papyrusurkunden etc., hrsg. v. E. Kiessling, I. Berlin 1925, 726, nicht bei Lampe, nicht im LBG.

${ }^{37} \mathrm{Vgl}$. LSJ 865b, s.v.

38 4Reg 18.21, Is 36.6, Ez 29.6.- Mat 11.7, 12.20, 27.48, Mar 15.19,36, Luk 7.24, 3Joh 13, Apok 11.1, 21.15.

39 Ruth 1.20 .

${ }^{40}$ Laut TLG insgesamt 17 Belege, davon 15 in als echt angesehenen Hymnen.

${ }^{41}$ So z.B. im Hymnus auf die Enthauptung des Täufers (MaAs / Trypanis 38.17.2f.):

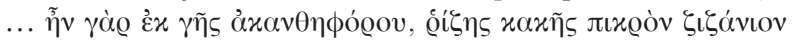

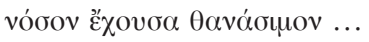




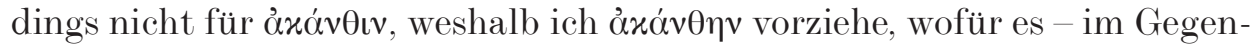
satz zu öxav $\theta \alpha v$ - zwar nur wenige Belege gibt ${ }^{42}$, doch entspricht es dem Metrum des Verses. Der inhaltliche Bezug zur Textstelle verstärkt sich, wenn man bedenkt, dass zahlreiche Distelarten genießbare, auch süße Wurzeln haben ${ }^{43}$. Obgleich das folgende Relativpronomen ô vielleicht auch auf den gesamten vorangehenden Satz bezogen werden kann ${ }^{44}$, ziehe ich daher aus Bedeutungsgründen vor, es durch ๆ̄ zu ersetzen. Text und Übersetzung könnten daher lauten:

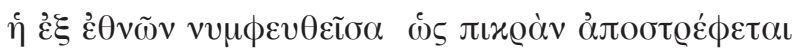

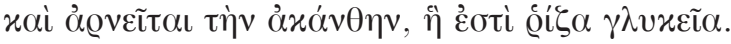

Die Vermählte aus heidnischem Stamm weist als bitter zurück und verweigert sich der Distel, die eine süße Wurzel ist.

\section{Auf die Samaritanerin (H. 19, Grosdidier de Matons, H. 9, Maas / Trypanis)}

Im selben Hymnus heisst es in der letzten Strophe ${ }^{45}$ in Codex Q (22.5):

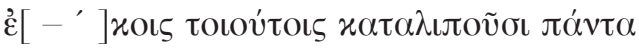

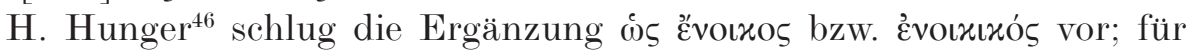
letzteres entschied sich auch Maisano ${ }^{47}$.

Mir erscheint hier die Ergänzung tenen Textbestand näher ist, als die beiden anderen Vorschläge und auch dem Metrum entspricht. Zudem ist દ̉ंi mit Dativ in entsprechender Bedeutung bei Romanos häufig belegt ${ }^{48}$. Die Übersetzung von Zeile 5ff. der Strophe könnte dann lauten:

Für solche Wohnungen werden sie alles zurücklassen,

Felder, Eltern und ihr Liebstes,

und ich werde ihr Gott sein und ihr Retter aus den Fallstricken,

sie aber mein geheiligtes Volk; ine Behausung werden sie machen

für die ewige und unteilbare

Dreifaltigkeit, die in Fülle dies verströmt:

Jubel und Erlösung!

${ }^{42}$ Chronologisch nahestehend Gregor von Nyssa, De anima et resurrectione, $P G$ 46.109D.

${ }^{43} \mathrm{Zu}$ den als $\alpha \ddot{x} \alpha v \theta \alpha$ bezeichneten Disteln vgl. LSJ $47 \mathrm{a}$, s. v.

${ }^{44}$ Vgl. hierzu E. Schwyzer, Griechische Grammatik, 2. Band: Syntax und syntaktische Stilistik (Handbuch der Altertumswiss. 2.1.2). München 1950, 639f.

45 Vgl. zur Stelle Grosdidier de Matons II 352f. A.1.

${ }^{46}$ In seinen Rezensionen in BZ 57 (1964) 441 und BZ 61 (1968) 94.

${ }^{47}$ Maisano I $270 \mathrm{f}$. und 79.

${ }^{48}$ H. 2.8.5, 5.22.9, 8.22.6, 26.17.2, 30.15.3, 34.19.6, 54.2.7. 


\section{Auf die Vermehrung der Brote (H. 24, Grosdidier de Matons, H. 13, Maas / Trypanis)}

In diesem Hymnus sorgen sich die Apostel um die Versorgung der Massen in der Wüste und sprechen, laut Codex Q (10.1-7):

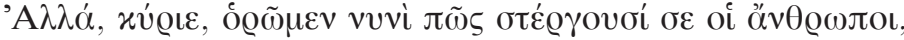

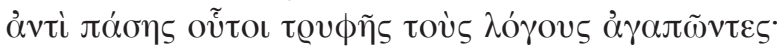

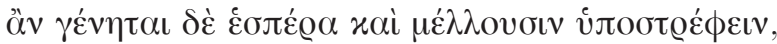

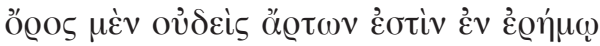

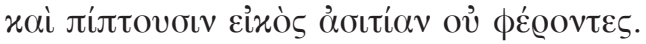

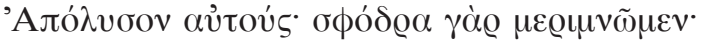

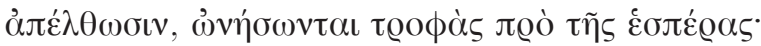

Hier ist in Z. 4 entweder őoos oder ov̉deís zu korrigieren. Da „,kein Berg

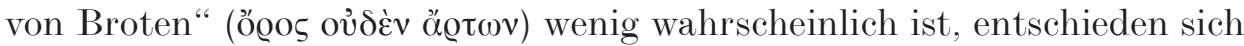

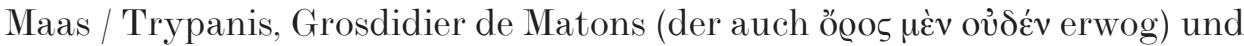
Maisano für лó@os, und Tomadakes für das paläographisch naheliegende őoos. (Man könnte eventuell auch тœóлos in Betracht ziehen, das zwei Strophen weiter, in 24.12.5 verwendet wird.)

Aus dem Zusammenhang bietet sich eher jógos an, da zwar nicht letz-

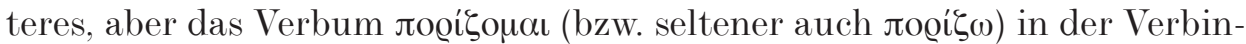

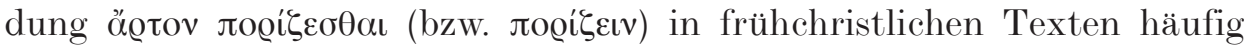
belegt ist ${ }^{49}$. Daraus ergibt sich die folgende Übersetzung dieser Verse:

Herr, wir sehen jetzt, wie sich die Menschen Dir zuwenden,

da sie anstatt jeglichen Vergnügens die Worte lieben.

Doch wenn es abend wird und sie heimkehren wollen,

finden sie keine Versorgung mit Broten in der Wüste

und fallen wahrscheinlich erschöpft nieder, da sie den Mangel nicht ertragen.

Entlasse sie! Wir sind gar sehr in Sorge:

So lass sie gehen, lass sie Nahrung kaufen vor dem Abend!

9. Auf die Auferweckung des Lazarus (H. 26, Grosdidier de Matons, H. 14, Maas / Trypanis)

In Handschrift Q fehlen in Oikos 3.9 zwei oder drei Silben ${ }^{50}$ :

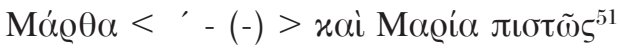

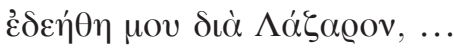

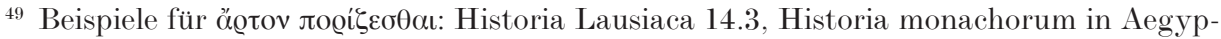

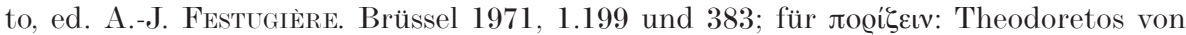
Kyrros, Comm. in Isaiam, ed. J.-N. Guinot, I (SC 276). Paris 1980, 13.391.

${ }^{50}$ Zum Metrum s. Grosdidier de Matons III 153. Vgl. im selben Hymnus die Oikoi 4.9 und 18.9 .

51 Vgl. Grosdidier de Matons III 158, app. crit. 
Wegen des Singulars $\dot{\varepsilon} \delta \varepsilon \eta \dot{\theta} \theta \eta$ in Z. 10 ist ein weiteres Verbum erforderlich. Maas und Trypanis ergänzen, unter Hinweis auf 26.2.8, '̌́c; Maisano folgte ihnen.

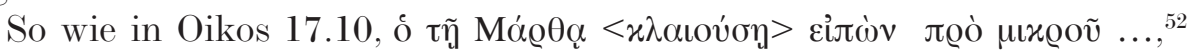
bietet sich eher die Ergänzung $<{ }^{\prime \prime} x \lambda \alpha \iota \varepsilon>a^{53}$, die auch deswegen nahe liegt,

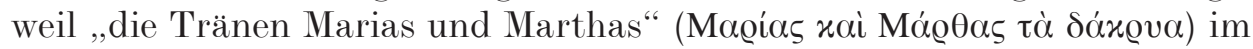
Prooimion des selben Hymnus angesprochen werden und im folgenden zweiten Hymnus auf Lazarus (27) den Refrain bilden. Somit ergibt sich folgende Übersetzung:

Martha weinte und Maria flehte gläubig zu mir um Lazarus willen, ...

\section{Auf die zehn Jungfrauen (H. 51, Grosdidier de Matons, H. 48, Maas / Trypanis)}

In Handschrift Q lautet das Ende von Oikos 3.10-12:

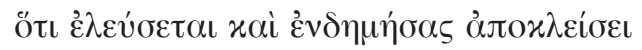

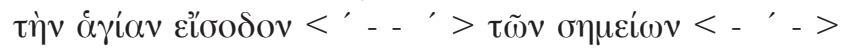

"Avoı $\xi o v$

Für die beiden Lücken in Z. 11sind folgende, inhaltlich und metrisch passende Ergänzungen denkbar ${ }^{54}$ :

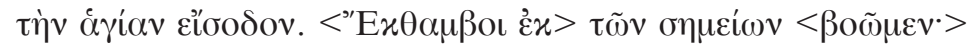

$\mathrm{Zu}$ हैx $x \propto \mu \beta$ o in ähnlicher Bedeutung bei Romanos vgl. die Hymnen 39

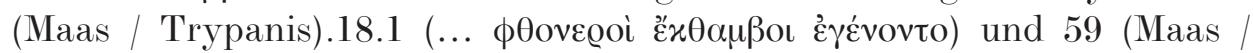

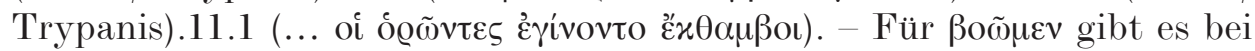
Romanos acht Belege $\mathrm{e}^{55}$.

Unter Berücksichtigung der Ergänzungsvorschläge lautet dann die Übersetzung:

... denn er wird kommen, wird Wohnung nehmen und dann

den heiligen Eingang verschließen. Aufgeschreckt von den Anzeichen lasset uns rufen:

Mach auf!

${ }^{52}$ A. KambYLis, Theol. Literaturzeitung 92 (1967) 284, schlägt an dieser Stelle die Ergänzung $\theta \varepsilon \ddot{x} \tilde{\omega} \varsigma$ vor.

${ }_{53}$ Zur Formulierung im Imperfekt vgl. - abgesehen von der Sinnhaftigkeit - auch H.

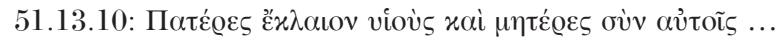

${ }^{54} \mathrm{Zu}$ anderen Ergänzungsvorschlägen von Pitra und Tomadakes vgl. Grosdidier de Matons V 302, app. crit.

${ }_{55}$ H. 9 pr.3, 41 pr.4, 51.4.11,10.11,11.11,16.11,17.11, 52pr.2, sowie Akathistos 17.5. 


\section{Auf die Neuerleuchteten (H. 52, Grosdidier de Matons, H. 53, Maas / Trypanis)}

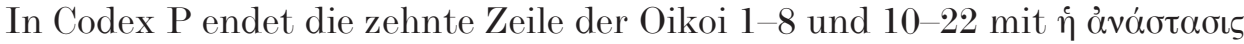

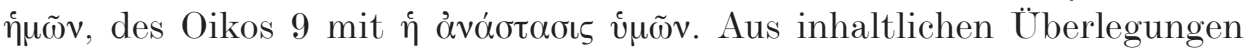

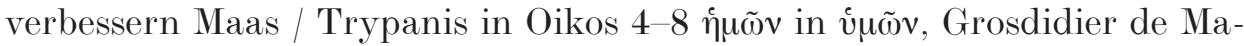

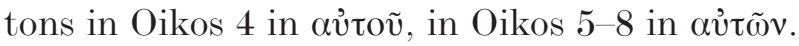

Zunächst ist festzuhalten, dass eine mit Unachtsamkeit des Schreibers

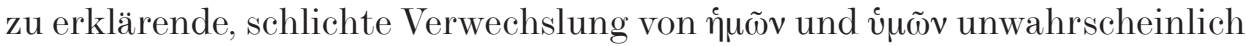

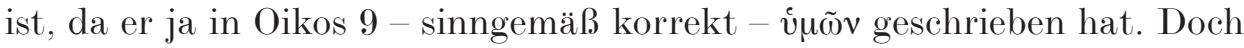
scheinen mir die betreffenden Textstellen überhaupt nicht verbesserungsbedürftig, da ich davon ausgehe, dass der Teufel in Oikos 4 lediglich die Zeilen 4-9 spricht und in den Zeilen 10-11 (Refrain) der Berichterstatter (der Chor) antwortet:

Und wer schenkte ihm dies? Gewiss unsere Auferstehung!

Ruhm, Ruhm sei Dir, dass Du daran Gefallen fandest!

In Oikos 5 ist eine analoge Interpretation naheliegend: In Zeile 1-7 ist der Teufel am Wort, ab Zeile 8 erfolgt die Antwort des Berichterstatters. In den Oikoi 6-8, in denen Vorbilder der Taufe im Alten Testament angeführt werden, spricht der Teufel nicht, doch kommt er dann in den Oikoi 9 und 10 noch einmal zu Wort. Ab hier ist auch die Übereinstimmung der Editionen mit Codex P wieder gegeben.

\section{Auf jedes Erdbeben und jede Feuerbrunst (H. 54, Grosdidier de Matons, H. 54, Maas / Trypanis)}

Der Dichter preist Justinians Neubau der Hagia Sophia und appelliert in Oikos 24 an die Gläubigen, Gott um Hilfe zu bitten und dadurch das Kaiserpaar zu erfreuen. 24.5-7 lauten im Codex Q:

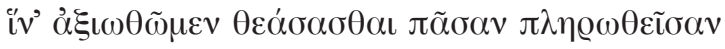

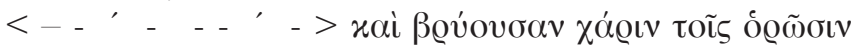

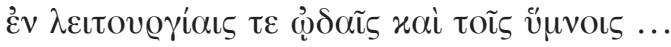

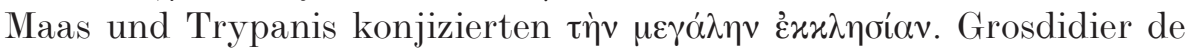
Matons erwägt mehrere Ergänzungsmöglichkeiten ${ }^{56}$, wobei er auch Bezüge zu Stellen des Alten Testaments prüft ${ }^{57}$, sich letztlich aber für keine der von ihm diskutierten Varianten entscheidet. Maisano wählte aus seinen Vor-

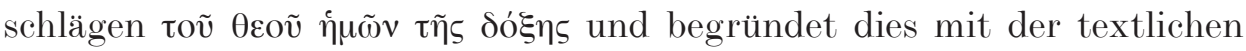

${ }^{56} \mathrm{Vgl}$. Grosdidier de Matons V 496f. mit app. crit. und A. 5.

57 Unter diesen scheinen mir freilich Is 6.1 und 1 Kge 8.11 kaum einen Bezug zum Hymnus zu haben, und Agg 2.9 zwar zum Kirchenbau zu passen, aber nicht zur konkreten Textstelle. 
Nähe zu 1Könige 8.11 ${ }^{58}$. Der Bezug scheint mir trotz der vergleichbaren Situation - Einweihung des salomonischen Tempels - zu allgemein. Daher

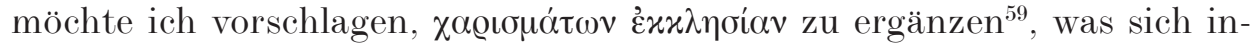
haltlich gut in den Kontext der Zeile fügt. Daraus ergibt sich die folgende Übersetzung:

... auf dass wir für würdig befunden werden zu schauen, wie jede Kirche erfüllt ist von Gnadengaben und für die Schauenden Gnade verströmt, in Gottesdiensten, Gesängen und Hymnen, ${ }^{60} \ldots$

\section{Ein Gebet an Christus und die Theotokos (H. 56, Grosdidier de Matons, H. 84, Maas / Trypanis)}

Das Gebet ist in einem einzigen Codex, dem Sabbaiticus 434, überliefert. Es enthält deutliche Anklänge an den Akathistos Hymnos. Da dieser nunmehr vor die Mitte des 5. Jahrhunderts zu datieren ist ${ }^{61}$, stellen diese Parallelen kein Argument gegen eine Zuweisung des Textes an Romanos dar, wenngleich die vorliegenden Argumente meiner Meinung nach derzeit keine endgültige Entscheidung zulassen ${ }^{62}$.

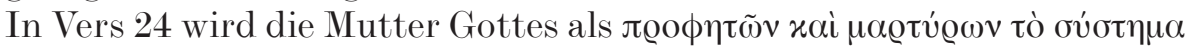
bezeichnet (,Zunft der Propheten und Märtyrer“), was in Bezug auf Maria

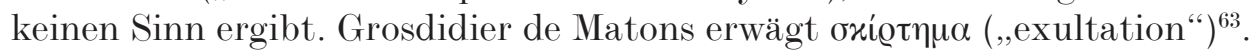

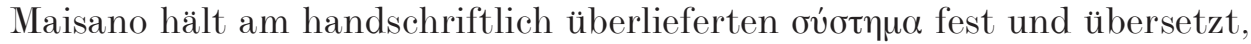
die unpassende Bedeutung mildernd, mit „,concordia dei profeti e dei mar-

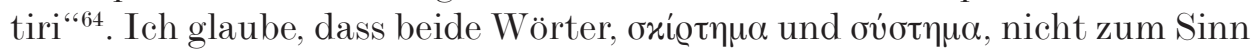
des folgenden Verses passen, und schlage daher - in Anlehnung an Akathi-

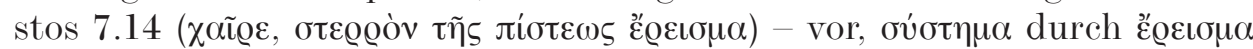
$\left(\right.$,Stütze“) zu ersetzen, was sinnvoll ist ${ }^{65}$. Daraus ergibt sich die Übersetzung von Vers 24f:

Stütze der Propheten und Märtyrer

und Hilfe der gesamten Welt!

58 Maisano II 470f. mit A. 52.

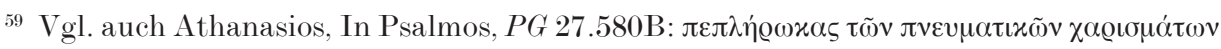

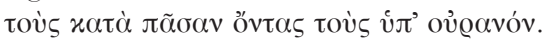

60 Z. 12 enthält eine Anspielung auf Epheser 5.19f.

${ }_{61}$ Vgl. L.-M. PeltomaA, Zur Interpretation des Hymnos Akathistos. Byzantium and the North / Acta Byz. Fennica 8 (1997) 97-106, und dies., The Image of the Virgin Mary in the Akathistos Hymn (The Medieval Mediterranean, 35). Leiden / Boston / Köln 2001, hier $113 f$.

62 Diskussion bei Grosdidier de Matons V $527 \mathrm{ff}$.

63 Grosdidier de Matons V 538, A.1.

64 Maisano II $570 \mathrm{f}$.

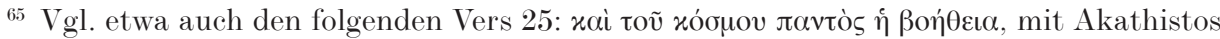

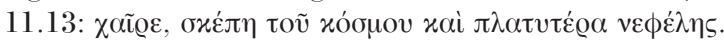




\section{Auf den von den Aposteln geheilten Lahmen (H. 60, Grosdidier de Matons, H. 39, Maas / Trypanis)}

Der Hymnus berichtet in Anlehnung an Kapitel 3 der Apostelgeschichte über die Wunderheilung des lahmen Bettlers am Tempeleingang in Jerusalem durch die Apostel Petrus und Johannes. Am Beginn von Oikos 8 spricht der Bettler die beiden Apostel an, bevor sie in den Tempel eintreten; er sagt (60/39.8.3-4) laut Codex P:

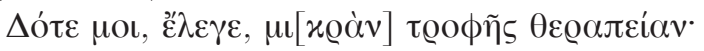

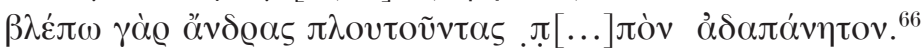

Die Textlücke, die im Codex wenig Raum beansprucht und aus metrischen Gründen eine unbetonte Silbe enthalten soll, ergänzte P. Colaclides ${ }^{67}$

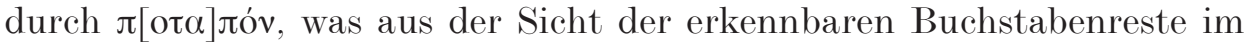
Codex gut passt, und auch vom inhaltlichen Zusammenhang her möglich ist $^{68}$, sich aber aus metrischen Gründen nicht empfiehlt.

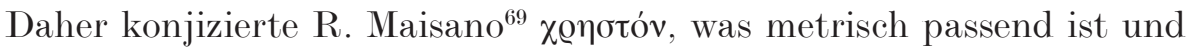
auch einen guten Sinn ergibt ${ }^{70}$, während die Ergänzung andererseits hinsichtlich des Buchstabenbestandes stärker vom Codex abweicht.

Durchsucht man den Wortbestand des Romanos auf oxytone zweisilbige Substantiva, die auf - $\pi$ óv enden, so bietet sich m. E. auch die Ergänzung $x[\alpha \varrho] \pi$ óv als „Frucht“ im weiteren Sinn, also „Gewinn“, „Ertrag“" $u$.ä. an, was metrisch passend ist und lediglich das - ohnedies unsichere $-\pi$ vor der Lücke durch $x$ ersetzt.

Vergleichbar ist bei Romanos z. B. eine Textstelle im Bußgebet, H. 55.13.14 :

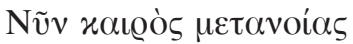

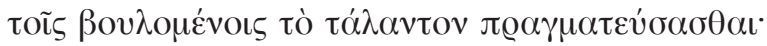

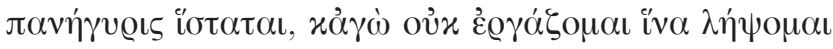

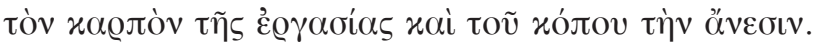

Jetzt ist die Zeit der Reue für die,

die mit ihrem Talent wuchern wollen. ${ }^{71}$

Das Fest ist ausgerichtet, doch ich arbeite nicht dafür,

den Ertrag der Arbeit und das Ende meiner Mühsal zu gewinnen.

${ }^{66}$ Zitiert nach MaAs / Trypanis 306 (Der Hymnus ist in den Sources Chrétiennes noch nicht ediert).

${ }^{67}$ P. Colaclides, Critical Note on a Line of Romanos. BZ 61 (1968) $268 \mathrm{f}$.

${ }^{68}$ Colaclides, a.O. 269, übersetzt: „for what abundantly wealthy men have I before me!"

69 Maisano II 144f., mit A. 14.

${ }^{70}$ Maisano, a.O., übersetzt: „uomini di una bontà inesauribile“.

${ }^{71}$ Mat 25.14-30. 
Für verwandte oder ähnliche Bedeutungen gibt es in den Werken der Kirchenväter zahlreiche Belege. Als Beispiel sei die Predigt des Johannes Chrysostomos über die Apostelgeschichte angeführt, in der in Kapitel 5 ein dem Hymnus verwandtes Thema behandelt wird, wie nämlich unbemittelte Menschen ihrer Pflicht, bedürftigen Armen zu helfen, nachkommen

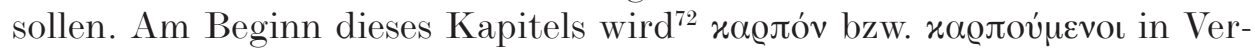

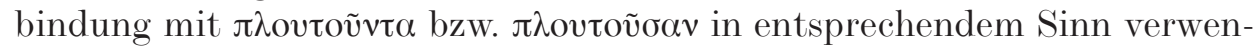
det.

Daher bietet sich die folgende Übersetzung der Verse 3-4 an:

Gebt mir eine kleine Spende für Essen!

Ich sehe, ihr Männer seid reich an unerschöpflichem Ertrag!

\section{Auf den von den Aposteln geheilten Lahmen (H. 60, Grosdidier de Matons, H. 39, Maas / Trypanis)}

Im selben Hymnus vollbringt Petrus unter Mitwirkung des Johannes das Wunder der Heilung des lahmen Bettlers (Oikos 17). Daraufhin fragen (Oikos 18) die in großer Zahl anwesenden Tempelbesucher Petrus, woher er die Gabe und den „unerschöpflichen“ (spirituellen) Reichtum habe, Heilungswunder zu wirken; die Strophe schließt (60/39.18.8-11) laut Codex P:

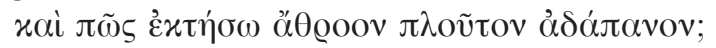

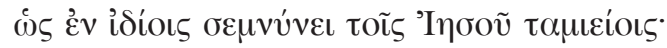

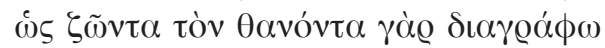

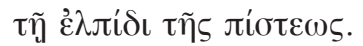

Da der Sinn der Zeilen 9 und 10 in der vorliegenden Textform nicht klar ist, schlägt P. Maas vor, die beiden Verba finita leicht zu ändern ${ }^{73}$, und zwar

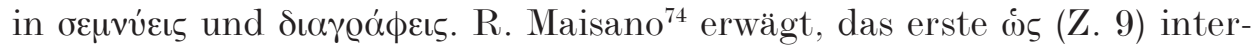
rogativ zu verstehen, also den ganzen Satz als eine Frage des Juden, der auch bisher gesprochen hat. Im anschließenden Vers (Z. 10) geht er bei $\delta\left\llcorner\alpha \gamma o \alpha ́ \phi \omega^{75}\right.$ von einer der "geläufigen " klassischen Bedeutungen aus, nämlich „durchstreichen“. Er übersetzt daher die Z. 9-11 folgendermaßen:

Come puoi pavoneggiarti con i tesori personali di Gesù?

Io escludo che colui che è morto sia vivente

per la speranza della fede!

Die Übersetzung hat den Vorteil, ohne Textveränderungen auszukommen. Meine Bedenken richten sich allgemein gegen den zweimaligen Wechsel der Bezugsperson in zwei Zeilen; während der Jude sonst in der gesam-

\footnotetext{
72 Johannes Chrysostomos, PG 60.170AB.

$73 \mathrm{Vgl}$. Maas / Trypanis 309, app. crit.

74 Maisano II 151, mit A. 30.

$75 \mathrm{Vgl}$. LSJ 392b und Lampe 346a.
} 
ten Strophe Petrus in der zweiten Person anspricht, wechselt er hier (für eine Zeile) in die dritte Person und anschließend in die erste Person.

Mir erscheint daher in Z. 9 aus dem Kontext ein minimaler Eingriff

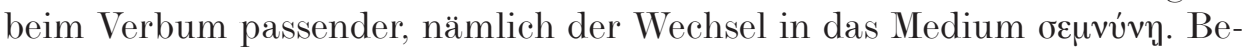

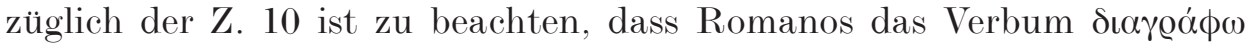
öfter verwendet und es an allen anderen Stellen ${ }^{76}$ eindeutig im Sinne von „beschreiben“ einsetzt. Daher ziehe ich diese Bedeutung vor, muss aber dann - wie P. Maas - von einer 2. Person ausgehen ${ }^{77}$. Daraus ergibt sich der folgende Übersetzungsvorschlag (Z. 8-11):

Und wie hast du plötzlich solch unerschöpflichen Reichtum erworben?

Mit Jesu Schätzen verherrlichst du dich wie mit deinen eigenen!

Als Lebenden beschreibst du ja den Verstorbenen

in der Hoffnung des Glaubens!

${ }^{76}$ H. 20.11.6, 22.5.2 und 50.5.8.

${ }^{77}$ Hierbei ist zu bemerken, dass nach Lampe 346a auch medialer Gebrauch des Verbums,

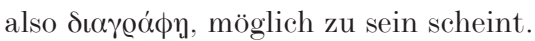

